\title{
The perceived benefits of the Maties Injury Programme among students
}

\author{
HLP Bates, ${ }^{1,2}$ B MedSci; JH Kirby, ${ }^{1}$ MB, ChB, MSc (Sports Med) \\ ${ }^{1}$ Stellenbosch University Centre for Human Performance Sciences \& Campus \\ Health Services, Stellenbosch, South Africa \\ ${ }^{2}$ College of Medical and Dental Sciences, University of Birmingham, \\ Birmingham, United Kingdom
}

Corresponding author: JH Kirby (jkirby@sun.ac.za)

\begin{abstract}
Objective: To investigate how athletes attending the Maties Injury Programme (MIP) at Stellenbosch University perceived the service, and to highlight areas they believed could be improved.

Participants: Thirty athletes representing Stellenbosch University attended the MIP following injury in May 2014.

Methods: A questionnaire-based service evaluation to assess patients' perceptions of the MIP.

Results: Ninety-three percent of participants indicated that they benefited from attending the injury clinic. Across all domains in the questionnaire patients reported high levels of satisfaction with the MIP. Four participants made suggestions for improvement, which have been considered.

Conclusions: The theoretical benefits of providing a specialised sports injury assessment as part of the Campus Health Services for athletes at Stellenbosch University have been supported by the encouraging responses of patients. Incorporating sports injury programmes into campus health services at more universities should be considered.
\end{abstract}

Keywords: service survey, high performance programme, injury clinic

S Afr J Sports Med 2016;28(2):36-38. DOI:10.17159/2078-516X/2016/v28i2a170

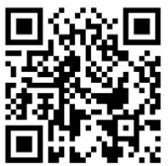

Doctors with experience and qualifications in identifying and treating sports injuries are best placed to establish which causative factors result in an injury and implement appropriate treatment. ${ }^{[1,2]}$ Many universities have therefore incorporated dedicated sports injury programmes into their campus health services. The Maties Injury Programme (MIP) at Stellenbosch University is one such an example.

The MIP offers free and rapid specialist assessment of sports injuries sustained during training or participation in university sporting activities. Assessing doctors can arrange for specialist referrals or further investigations. The costs are apportioned between Maties Sport and the Stellenbosch University's Campus Health Services. The programme aims to provide a holistic approach to care by assessing the injury; providing information to the patients and an agreed tailored treatment and rehabilitation plan with the sportsperson, in addition to providing training adaption advice for coaches.

There is little available literature documenting the perceived benefits of involving sports injury specialists in campus health services. Similar constructs to the MIP are available in the United States; however, no research could be identified in assessing patient perceptions. Moreover, there is little published research investigating the perceptions of patients attending sports physicians, although it is possible that unpublished evaluations conducted within organisations may exist.

Appropriate patient support from the healthcare team can improve outcomes of sports injuries. ${ }^{[1,2]}$ In sport, the use of a biopsychosocial patient assessment model with effective communication between the healthcare team and patient is vital to deal with the stresses and social pressures of competition. ${ }^{[2]}$ Successful communication and high levels of patient satisfaction are likely to contribute to better outcomes in injured patients.

As well as good communication, understanding of the complexity of sports medicine is key in injury management at all levels. Vergeer suggests family practitioners are often unable to deal satisfactorily with sports injuries because of insufficient training. ${ }^{[3]}$ For elite varsity athletes, assessment by a sports physician is crucial to achieving overall optimal sports performance. ${ }^{[2]}$ Thorough knowledge of sports injuries is particularly important for treating first-year university athletes when dramatic increases in training intensity may result in chronic injuries. Cooperation between healthcare professionals and coaches is essential to adapt programmes to meet individual needs, as well as to identify and address the social and psychological factors accompanying injuries. ${ }^{[2]}$

The volume of supportive literature on the objective benefits of sports injury clinics suggest investigation of perceived (as opposed to objective) benefit is unnecessary. However, conducting a service evaluation to assist in assessing the success of the programme, and investigating the possibility that the MIP and the communication of information to coaches could be perceived as a benefit to the team rather than to the individual. The secondary aim arose from concerns about the detrimental effect of such a perception on the relationship between the athlete and team doctor in elite sport. ${ }^{[4]}$

The aim of this questionnaire-based service evaluation was to investigate how the athletes attending the MIP perceived the service and to highlight areas they believed could be improved.

\section{Methods}

A questionnaire-based service evaluation was used to assess patients' perceptions of the MIP over four weeks in May 2014. The study received written approval from the Directors of Maties Sport, Campus Health Services and Centre for Human Performance Sciences, University of Stellenbosch.

There was no available validated questionnaire to assess patients' perceptions of benefits from a sports injury programme. Therefore this study used seven questions based on the 'Medical Interview Satisfaction Scale'; ${ }^{[5]}$ a self-report questionnaire using a five-point Likert scale that has been validated in South Africa. The questionnaire was adapted to measure satisfaction with treatment from athletic trainers in injured athletes from previous research by replacing the word 'illness' with 'injury ${ }^{[5]}$ (Appendix 1).

Demographic data and injury type were recorded. Patients were also asked to rate the perceived benefit from attending the clinic on a fivepoint Likert scale, to identify the most beneficial aspect of attending the injury clinic and to suggest improvements. The questionnaire was piloted on three MIP patients. No misunderstandings of the questions were noted. Pilot responses were not included in the analysis. 
Results were presented using descriptive statistics. The proportion of patients from each sport and mean age were assessed, as well as the distribution of traumatic and non-traumatic causes of injury. Responses for each of the eight questions were analysed using a mean average calculation and standard deviation. Qualitative responses were not sufficiently $\mathbb{a}$ bstantial $\delta \mathrm{r}$ if $\mathrm{ll}$ analysis but were grouped by theme and presented quantitatively.

\section{Results}

Thirty-three students attending the MIP were approached - three declined to participate. All patients ( $\mathrm{n}=21$ males, nine females) saw a qualified sports doctor. The mean age of the students was 21 years (range: 18-32 years). Rugby accounted for the largest proportion of injuries seen $(40 \%, n=12)$ with hockey injuries the second most common $(27 \%, n=8)$. The o ther i njuries w ere $\mathrm{f}$ rom $\mathrm{s}$ occer $(\mathrm{n}=4)$, athletics $(\mathrm{n}=3)$, ultimate Frisbee $(\mathrm{n}=1)$, badminton $(\mathrm{n}=1)$ and water polo $(\mathrm{n}=1)$. Non-traumatic injuries accounted for $57 \%$ of all injuries $(n=17)$.

Thirty responses were collected, with only one questionnaire incomplete. Figure 1 shows the distribution of the scale rating for each question and the mean response value with standard deviations. None of the participants 'strongly disagreed' with any of the statements. The majority of responses (82\%) were positive ('agree' or 'strongly agree'). Only two statements did not have a mean score value corresponding with a positive response. Statement 6: 'After talking to the doctor I felt much better about my problems' received the lowest average rating $(3.8 \pm 0.7)$ and Statement 5: 'The doctor has relieved my worries about being seriously injured' had a mean rating of (3.9 \pm 0.9$)$. The final statement 'Overall I have benefitted from attending the sports injury clinic' was the highest scored statement ( $4.6 \pm 0.6)$ with $93 \%$ $(n=28)$ of responses either 'agree' or 'strongly agree' and $7 \%(\mathrm{n}=2)$ responding 'uncertain'.

Twenty-four participants identified the aspect of the injury programme that they felt was most beneficial. F ive $t$ hemes w ere identified: information about and assessment of an injury; rapid referral to specialist care; emotional support; convenience; and treatment. Eighty three percent $(n=20)$ of those that responded felt that the information about and the assessment of their injury were the most beneficial aspects of the injury programme.

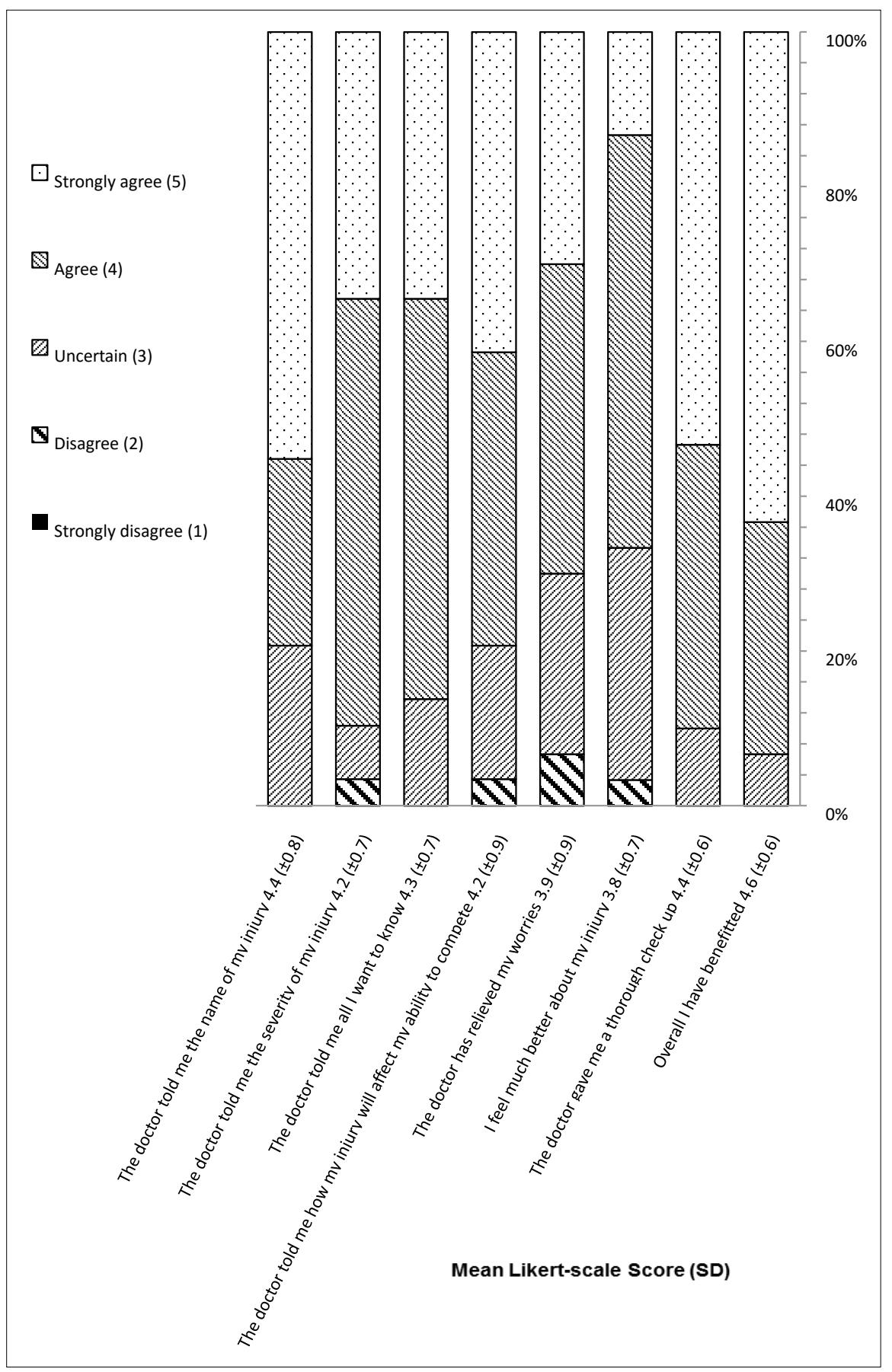

Fig. 1: Perception of benefit Likert responses

Four participants provided suggestions for improving the MIP. The suggestions were: provision of an estimated duration of rehabilitation; funding of investigations; staff should be friendlier; and extension of clinic hours during competition times.

\section{Discussion}

Most participants (93\%) agreed that they had benefited from attending the injury clinic with the remainder indicating 'uncertain'. Most responses (82\%) were 'agree' or 'strongly agree' for all the statements in the question- naire, indicating a consistently positive perception of the MIP.

Overall, the participants reported high levels of satisfaction with the MIP; in particular, the identification of the injury and its severity, its impact on the competition and the answering of queries. Lowest rated statements pertained to the doctor's impact on a patient's emotional response to their injury. As the questionnaire was completed following the initial consultation, patients may have received disappointing news with regard to their injury assessment and the 
lower scores for the Statements Five and Six can be attributed to the variable nature of the results of the injury assessment. Repeating the questionnaire after a patient's recovery may result in changes of these scores.

The encouraging responses of the participants in this study support the theoretical benefits of a dedicated sports injury programme within campus health services generally at Stellenbosch University, as well as the continued provision of the MIP. The consistent agreement with statements relating to communication of information suggests that holistic care is being provided. ${ }^{[1]}$ The high level of patient satisfaction and the impressive coordination between healthcare professionals, coaches, managers and players make this programme a potential model for other universities. None of the responses commented on coaching or team influence in the evaluation of the MIP - refuting Furrow's suggestion that injured sportspeople may feel sports doctors are provided primarily for the coach's benefit. ${ }^{[4]}$

The results indicate that the programme is seen as beneficial by the majority of patients and that the assessment and subsequent information provided is perceived as very valuable. This study also provided an avenue for patients to suggest changes which have been reported to the MIP.

\section{Limitations}

Based on the experiences at the MIP it can be observed that that younger players commonly present with non-traumatic injuries from the sudden increase in training as they move from school to university. Many such injuries may have been missed due to the short duration of the study and its timing in the academic year. As these patients form a large proportion of MIP patients, assessment of their perceptions following their initial assessment during and after rehabilitation, is an avenue for further research.

There are inherent problems with questionnaire studies in general. ${ }^{[6]}$ This questionnaire was limited by the inclusion of questions that were likely to be disadvantageous in the consultation - rewording or removal of these in future studies would be beneficial. Incorporation of interviews and focus groups could also be considered. ${ }^{[6]}$

\section{Conclusions}

Despite the limitations of the study, it has provided useful preliminary data on the perceptions of students attending the MIP, showing that it is regarded as beneficial by students. Overall, this study has suggested that the patients attending the MIP appreciate the rapid assessment and management advice given. The study has also shown that conducting service evaluations of the perceived benefits of existing sports injury programmes provides useful information both to health services and sports financers. The findings also add weight to the argument for the incorporation of more sports injury programmes in campus health services.

\section{References}

1. MacAuley D, ed. Oxford Handbook of Sport and Exercise Medicine. 2nd ed. London: Oxford University Press, 2013:35-63. ISBN: 9780199660155. [DOI: 10.1093/med/9780199660155.001.0001]

2. Stanish WD, Van Aarsen M, Evans NA. The modernday team physician: roles, responsibilities, and required qualifications. In: Micheli L, Pigozzi F, Chan KM et al., eds. Team Physician Manual: International Federation of Sports Medicine (FIMS). 3rd ed. New York: Routledge; 2013:3-9. ISBN: 9780415505338 [DOI: 10.1179/1753614613Z.00000000050]

3. Vergeer I. Family physicians and sports-injury care: Perceptions of coaches. Can Fam Physician 1997;43:1755-1761. [PMID: 2255428]

4. Furrow B. The problem of the sports doctor: Serving two (or is it three or four?) masters. St. Louis University Law J 2005;50:165-183.

5. Wolf MH, Putnam SM, James SA, et al. The Medical Interview Satisfaction Scale: development of a scale to measure patient perceptions of physician behaviour. J Behav Med 1978;1(4):391-40. [DOI: 10.1007/BF00846695]

6. Bowling A. Research Methods in Health: Investigating Health and Health Services. Buckingham, United Kingdom: Open University Press; 1997:242-357. ISBN-10 0335206433 [DOI: 10.1002/sim.755]

\section{Appendix 1. Questionnaire used to assess patients' perceptions of benefit from the sports injury programme}

Gender: M/F (Please circle) Age: Sport:
Cause of injury: Traumatic / Non-traumatic

Please circle your response to each question.

\begin{tabular}{|c|c|c|c|c|c|}
\hline & $\begin{array}{l}\text { Strongly } \\
\text { disagree }\end{array}$ & Disagree & Uncertain & Agree & $\begin{array}{c}\begin{array}{c}\text { Strongly } \\
\text { agree }\end{array} \\
\end{array}$ \\
\hline 1. The doctor told me the name of my injury in words that I could understand & 1 & 2 & 3 & 4 & 5 \\
\hline 2. After talking to the doctor, I know just how serious my injury is & 1 & 2 & 3 & 4 & 5 \\
\hline 3. The doctor told me all I wanted to know about my injury & 1 & 2 & 3 & 4 & 5 \\
\hline 4. The doctor told me how being injured will affect my ability to compete & 1 & 2 & 3 & 4 & 5 \\
\hline 5. The doctor has relieved my worries about being seriously injured & 1 & 2 & 3 & 4 & 5 \\
\hline 6. After talking to the doctor I felt much better about my problems & 1 & 2 & 3 & 4 & 5 \\
\hline 7. The doctor gave me a thorough check up & 1 & 2 & 3 & 4 & 5 \\
\hline 8. Overall I have benefitted from attending the sports injury clinic today & 1 & 2 & 3 & 4 & 5 \\
\hline
\end{tabular}

What was the most beneficial part of your attendance at the clinic?

Is there anything you think could be improved about the injury program at the clinic?

Any other comments:

Thank you for completing this survey. 